\title{
EDITORIAL
}

\section{Recognising "painless" heart attacks}

\section{C-K Wong, H D White}

\section{Heart attacks without chest pain all too often go unrecognised and untreated}

schaemic chest pain has been the hallmark of the clinical presentation of patients with acute coronary syndromes. New modalities for early diagnosis and treatment have been developed (for example, rapid troponin assays, glycoprotein IIb/IIIa receptor antagonists, anticoagulants, and facilitated percutaneous intervention), and cardiologists are now extending their reach to the emergency department-the portal of the hospital-in order to ensure that reperfusion and adjunctive treatments are instituted with minimal delay. ${ }^{1}$ Yet there has been relatively little evaluation of modern treatments in the context of "silent" myocardial infarction, a well recognised entity described by Herrick in $1912^{2}$ and documented further in long term follow up of the Framingham Study.

A recent issue of Heart featured the results of the EMMACE study, an audit of patient records from 20 adjacent hospitals in the Yorkshire region over a three month period in the mid 1990s. ${ }^{4}$ The authors identified 3684 consecutive cases with a possible diagnosis of acute myocardial infarction based on coronary care registers, clinical coding, and cardiac enzyme test results. The diagnosis was confirmed in 2096 patients, 20\% of whom had presented without chest pain. This proportion is somewhat higher than has been reported in previous studies, ${ }^{56}$ a finding the authors attribute to their retrospective methodology based on the final diagnosis of myocardial infarction, allowing identification of infarctions that were unexpected at presentation. ${ }^{4}$ In other words, many patients with "painless" infarction had an unexpected and often delayed diagnosis.

The patients who presented without chest pain were older (mean age $76.6 v 69.1$ years) and were more likely to be female $(54.6 \% v 35.3 \%)$ or to have a history of heart failure $(61.0 \% v 49.2 \%)$. They also had worse baseline haemodynamics and more severe left ventricular impairment than those who presented with chest pain. Even after adjustment for age and sex, though, they tended to receive suboptimal care-for example, only $39 \%$ spent any time in a coronary care unit, compared with $77 \%$ of patients with chest pain. By today's standards, the mortality rates at 30 days were high $-49.2 \%$ in patients without chest pain and $17.9 \%$ in those with chest pain-and the difference between the two groups persisted for up to two years. Importantly, presentation without chest pain was found to be independently associated with a $60 \%$ increase in the risk of death, after adjustment for other prognostic covariates including age, haemodynamics and left ventricular

Zealand;

harveyw@adhb.govt.nz impairment. Patients who presented without chest pain were also less likely to be discharged on aspirin (15.8\% $v 4.6 \%)$ or $\beta$ blocker treatment $(45.5 \% \vee 17.2 \%)$, and had lower participation rates in rehabilitation programmes and fewer follow up consultations.

\section{SILENT MYOCARDIAL ISCHAEMIA}

Some studies have reported high rates of silent ischaemia $(70-90 \%)$ in patients with angina undergoing Holter monitoring, but these rates may have been overestimated in some cases because of under reporting of anginal symptoms in patient diaries. ${ }^{7}$ In a study using a microprocessor that continuously analysed ambulatory electrocardiographic recordings and emitted an audible tone whenever ST segment depression was detected, ischaemic episodes occurring during normal daily activities were found to be "silent" in $53 \%$ of instances. ${ }^{7}$

Regardless of whether ischaemia is caused by increased myocardial demands (for example, during exercise testing $)^{8}$ or a reduced blood supply (for example, during balloon occlusion at angioplasty), wall motion abnormalities develop first, followed by ST segment changes and then anginal pain (if it occurs).

Silent ischaemia in asymptomatic patients has been shown to have negative prognostic implications, but can be reduced by atenolol treatment. ${ }^{10}$ The ACIP study screened 1959 patients with coronary artery disease using 48 hour Holter monitoring. Silent ischaemia was detected in 982 patients (49\%), and 618 of these were randomised to undergo either angina guided medical treatment, ischaemia (Holter) guided medical treatment, or revascularisation procedures. ${ }^{11}$ Most of these patients were men, with a mean age of 61 years, and many had multivessel disease (two vessels in $35 \%$ and three vessels in $41 \%$ ) and a normal left ventricular ejection fraction despite a history of prior myocardial infarction in $40 \%$. However, they were not completely asymptomatic, as $60 \%$ had had angina within six weeks of enrollment. Follow up at one year showed that the patients who had undergone revascularisation procedures had less asymptomatic ischaemia and a substantially lower rate of coronary events (death/myocardial infarction/non-protocol revascularisation/ readmission to hospital) than those managed according to the angina guided or ischaemia guided medical strategies ( $18 \% \vee 32 \% \vee 31 \%$,

Abbreviations: ACIP, asymptomatic cardiac ischemia pilot; EMMACE, evaluation of methods and management of acute coronary events 
respectively; $\mathrm{p}=0.003) .{ }^{12}$ Treadmill testing at eight weeks showed that patients who had undergone surgical revascularisation had less ischaemia than those revascularised by angioplasty, ${ }^{13}$ perhaps reflecting more complete revascularisation.

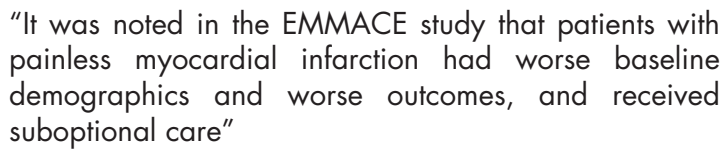

The relation between silent ambulatory ischaemia and painless myocardial infarction has not been evaluated in detail. It was noted in the EMMACE study that patients with painless myocardial infarction had worse baseline demographics. ${ }^{4}$ Almost one sixth of the patients were diabetic, and, as would be expected, ${ }^{14}$ they had a higher incidence of painless infarction than non-diabetics. It is not known what percentage of patients had impaired glucose tolerance. Diabetics are known to have a higher incidence of multivessel disease than non-diabetics, and tend to have poorer outcomes. ${ }^{15}$

\section{IS EARLY DIAGNOSIS OF PAINLESS INFARCTION A REALISTIC GOAL?}

During 30 year follow up of 5127 participants in the Framingham study, 708 myocardial infarctions were detected on routine biennial ECGs. More than 25\% of these went undiagnosed at the time of the event, and the proportion was higher in elderly patients and in women. Half of these events were virtually asymptomatic, and half were accompanied by atypical symptoms. The EMMACE study has extended the Framingham findings to patients admitted to hospital with "painless" myocardial infarction. ${ }^{4}$ About a third presented with shortness of breath, a third had symptoms suggestive of a cardiac problem (for example, collapse, upper body discomfort or resuscitated cardiac arrest), and a third had seemingly non-cardiac complaints (for example, non-specific unwellness or neuropsychiatric symptoms). The sex distribution of the presenting symptoms was not reported, and nor were concomitant illnesses. It is quite possible that these patients would have been diagnosed earlier had they routinely undergone serial ECGs and creatine kinase-MB or troponin tests. However, while the total number of patients presenting with similar symptoms during the three month study was not reported, it is likely that the numbers were substantial, raising the issue of cost effectiveness if such a strategy were implemented.

\section{The presenting ECG}

Patients with silent infarction may present with a nondiagnostic ECG - for example, left bundle branch block. The fibrinolytic therapy trialists' collaborative overview of patients presenting with typical symptoms of cardiac ischaemia confirmed that thrombolytic treatment was beneficial in those with bundle branch block. ${ }^{16}$ Patients with bundle branch block had the highest mortality rates at 35 days (18.7\% in those given thrombolytics $v 23.6 \%$ in those not given thrombolytics). The EMMACE study did not report the proportion of patients with left bundle branch block and their outcomes.

\section{Troponin concentrations}

The observed frequency of silent infarction will increase with the growing usage of troponin tests to diagnose myocardial infarction. Troponin concentrations remain raised for up to 14 days after myocyte injury, permitting a "delayed" diagnosis of myocardial infarction. Troponin testing may therefore be particularly useful for diagnosing painless myocardial infarction, as these patients generally present late.
When interpreting troponin tests, it should be borne in mind that while raised concentrations indicate myocyte damage, this can also be caused by factors other than myocardial ischaemia resulting from coronary thrombosis. In the absence of a history of ischaemia, other causes needed to be ruled out, such as renal disease, stroke, subarachnoid haemorrhage, pulmonary embolism, myocarditis, and heart failure. ${ }^{17-21}$

A study from the Cleveland Clinic prospectively evaluated the utility of troponin $\mathrm{T}$ and troponin I concentrations for predicting in-hospital and six month outcomes in 153 patients with suspected acute coronary syndromes, including 51 with and 102 without renal impairment (mean creatinine $3.83 \mathrm{mg}$ / $\mathrm{dl}$ and $1.12 \mathrm{mg} / \mathrm{dl}$, respectively). The sensitivity and specificity of troponins $\mathrm{T}$ and $\mathrm{I}$ in predicting adverse outcomes were found to be reduced in patients with renal disease. ${ }^{17}$ The EMMACE study did not report creatinine concentrations, but it is likely that they were higher in patients with painless acute myoardial infarction because they were older.

"In patients with ST segment elevation but no chest discomfort, the decision as to whether to administer thrombolytic treatment or not is a difficult one"

\section{MANAGEMENT}

Patients with painless myocardial infarction should be treated according to standard guidelines. In patients with ST segment elevation but no chest discomfort, the decision as to whether to administer thrombolytic treatment or not is a difficult one, as the duration of ischaemic symptoms is unclear. All patients should receive aspirin and $\beta$ blockers unless contraindicated, and patients with heart failure or impaired left ventricular function should be given ACE inhibitors. In those without ST segment elevation, aspirin and heparin should be given as standard treatment.

The roles of glycoprotein IIb/IIIa inhibitors and revascularisation have not been assessed in patients with painless infarction. However, the use of such treatments is recommended in patients with electrocardiographic evidence of ischaemia and raised troponin concentrations. ${ }^{22}{ }^{23}$ Treatment with abciximab has been shown to reduce ST segment shifts. ${ }^{24}$ In patients who are stable, tests for inducible ischaemia should be performed for the purpose of risk stratification, and continuous ambulatory ST segment monitoring can provide further information. ${ }^{25}$ Patients identified as having recurrent ischaemia after infarction (whether spontaneous, induced, silent or symptomatic) should undergo angiography with a view to revascularisation, as this strategy has been shown to result in better outcomes. ${ }^{26}$

The EMMACE study has highlighted the fact that presentation with painless myocardial infarction is more common than many of us realise. This is an important finding because "painless" myocardial infarction all too often goes unrecognised by both patients and physicians, leading to inferior care and poorer outcomes. These patients are at high risk of unpredictable ischaemic events, and have much to gain from the aggressive use of evidence based treatments.

\section{Authors' affiliations}

C-K Wong, H D White, Cardiovascular Research Unit, Green Lane Hospital, Private Bag 92 189, Auckland 1030, New Zealand

\section{REFERENCES}

1 White HD. Moving cardiology to the front of the hospital [editorial]. Heart 2000:84:573-4.

2 Herrick JB. Clinical features of sudden obstruction of the coronary arteries. JAMA 1912;59:2015-20.

3 Kannel WB, Abbott RD. Incidence and prognosis of unrecognized myocardial infarction: an update on the Framingham study. N Engl J Med 1984;311:1144-7. 
4 Dorsch MF, Lawrance RA, Sapsford RJ, et al. Poor prognosis of patients presenting with symptomatic myocardial infarction but without chest pain Heart $2001 ; 86: 494-8$

5 Goldstein RE, Boccuzzi SJ, Cruess D. Prognosis after hospitalization for acute myocardial infarction not accompanied by typical ischemic chest pain. The multicenter diltiazem postinfarction trial research group. Am J Med 1995;99:123-31

6 Herlitz J, Karlson BW, Richter A, et al. Prognosis for patients with initially suspected acute myocardial infarction in relation to presence of chest pain. Clin Cardiol 1992;15:570-6

7 Freedman SB, Wong CK. Triggers of daily life ischaemia. Heart 1998;80:489-92.

8 Heller GV, Ahmed I, Tilkemeier PL, et al. Comparison of chest pain, electrocardiographic changes and thallium-201 scintigraphy during varying exercise intensities in men with stable angina pectoris. Am J Cardiol 1991;68:569-74.

9 Hauser AM, Gangadharan V, Ramos RG, et al. Sequence of mechanical, electrocardiographic and clinical effects of repeated coronary artery occlusion in human beings: echocardiographic observations during coronary angioplasty. J Am Coll Cardiol 1985:5:193-7.

10 Pepine CJ, Cohn PF, Deedwania PC, et al. Effects of treatment on outcome in mildly symptomatic patients with ischemia during daily life: the atenolol silent ischemia study (ASIST). Circulation 1994;90 762-8

11 Pepine CJ, Geller NL, Knatterud GL, et al. The asymptomatic cardiac ischemia pilot (ACIP) study: design of a randomized clinical trial, baseline data and implications for a long-term outcome trial [published erratum appears in J Am Coll Cardiol 1995;26:842]. J Am Coll Cardio 1994;24:1-10.

12 Rogers WJ, Bourassa MG, Andrews TC, et al. Asymptomatic cardiac ischemia pilot (ACIP) study: outcome at 1 year for patients with asymptomatic cardiac ischemia randomized to medical therapy or revascularization. The ACIP investigators. J Am Coll Cardiol 1995:26:594-605.

13 Bourassa MG, Pepine CJ, Forman SA, et al. Asymptomatic cardiac ischemia pilot (ACIP) study: effects of coronary angioplasty and coronary artery bypass graft surgery on recurrent angina and ischemia. The ACIP investigators. J Am Coll Cardiol 1995;26:606-14.

14 Kannel WB. Lipids, diabetes, and coronary heart disease: insights from the Framingham study. Am Heart J 1985;110:1100-7.
15 Granger CB, Califf RM, Young S, et al. Outcome of patients with diabetes mellitus and acute myocardial infarction treated with thrombolytic agents. The thrombolysis and angioplasty in myocardial infarction (TAMI) study group. J Am Coll Cardiol 1993;21:920-5.

16 Fibrinolytic Therapy Trialists' (FTT) Collaborative Group. Indications for fibrinolytic therapy in suspected acute myocardial infarction: collaborative overview of early mortality and major morbidity results from all randomised trials of more than 1000 patients. Lancet 1994;343:311-22.

17 Van Lente F, McErlean ES, Deluca SA, et al. Ability of troponins to predict adverse outcomes in patients with renal insufficiency and suspected acute coronary syndromes: a case-matched study. J Am Coll Cardiol 1999.33:471-8.

18 James P, Ellis CJ, Whitlock RM, et al. Relation between troponin $T$ concentration and mortality in patients presenting with an acute stroke: observational study. BM 2000;320:1502-4.

19 Norris JW, Hachinski VC, Myers MG, et al. Serum cardiac enzymes in stroke. Stroke 1979:10:548-53.

20 Giannitsis E, Muller-Bardorff M, Kurowski V, et al. Independent prognostic value of cardiac troponin $T$ in patients with confirmed pulmonary embolism. Circulation 2000;102:211-7.

21 Missov E, Calzolari C, Pau B. Circulating cardiac troponin I in severe congestive heart failure. Circulation 1997;96:2953-8.

22 Bertrand ME, Simoons ML, Fox KAA, et al. Management of acute coronary syndromes: acute coronary syndromes without persistent ST segment elevation: recommendations of the task force of the European Society of Cardiology. Eur Heart J 2000;21:1406-32.

23 Cannon CP, Weintraub WS, Demopoulos LA, et al. Comparison of early invasive and conservative strategies in patients with unstable coronary syndromes treated with the glycoprotein Ilb/Illa inhibitor tirofiban. N Eng Med 2001:344:1879-87.

24 Klootwijk P, Meij S, Melkert R, et al. Reduction of recurrent ischemia with abciximab during continuous ECG-ischemia monitoring in patients with unstable angina refractory to standard treatment (CAPTURE). Circulation 1998;98:1358-64.

25 Larsson $\mathbf{H}$, Areskog M, Areskog NH, et al. The diagnostic and prognostic importance of ambulatory ST recording compared to a predischarge exercise test after an episode of unstable angina or non-Q wave myocardial infarction. Eur Heart J 1995; 16:888-93.

26 Madsen JK, Grande P, Saunamäki K, et al. Danish multicenter randomized study of invasive versus conservative treatment in patients with inducible ischemia after thrombolysis in acute myocardial infarction (DANAMI). Circulation 1997;96:748-55

\section{STAMPS IN CARDIOLOGY}

\section{Cardiac risk factors}

$\mathrm{H}$

ealthy eating, physical exercise, and routine medical examinaions have all been promoted on stamps. The 1994 stamp from Israel promoting healthy eating was part of a set of three stamps advertising "health and well-being", the other two stamps covering regular exercise and non-smoking. Physical fitness was the theme of the 1983 stamp from the USA showing joggers superimposed on an
ECG tracing. Community health and routine medical checks were the subject for the 41 cent Australian stamp from 1990 where heart and breast diseases were emphasised in the design of the stamp.

M K Davies A Hollman

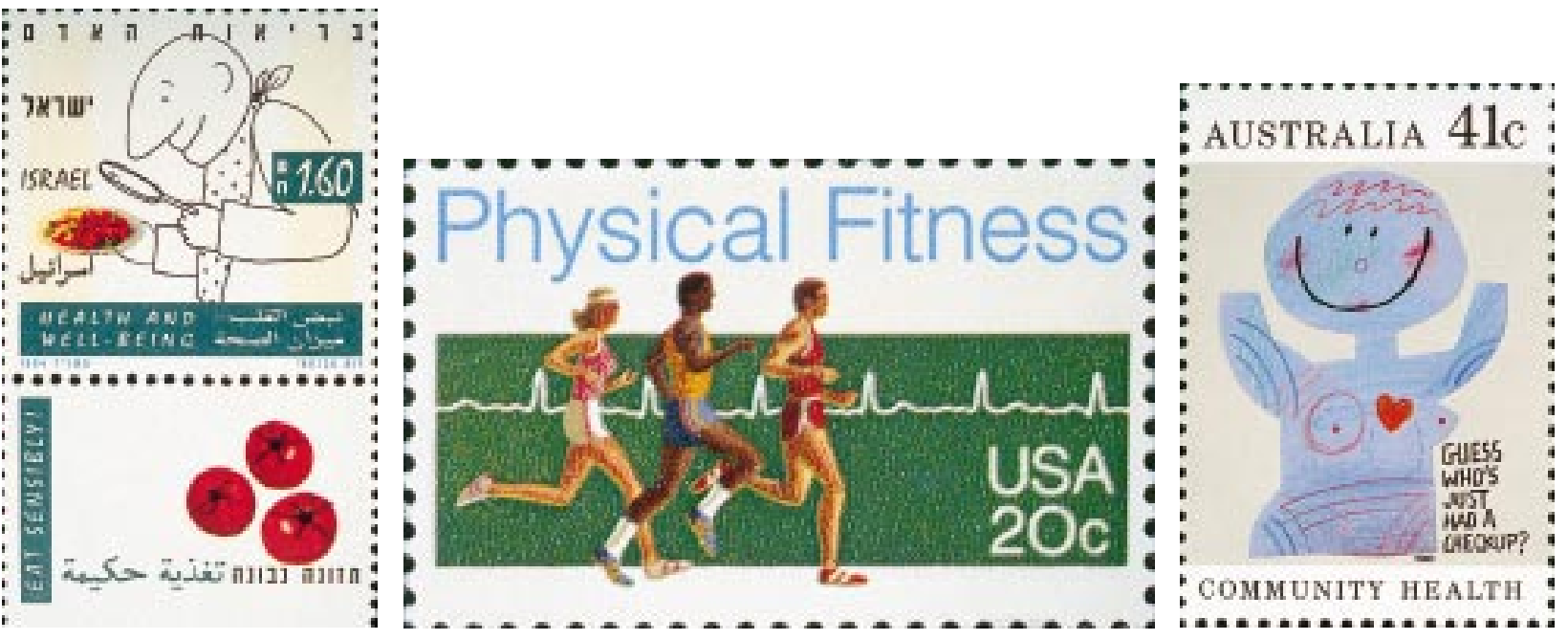

